\title{
Portafolios digital y evaluación de las competencias transversales en las prácticas externas del grado de Pedagogía de la Universidad de Barcelona
}

\author{
Assumpta Aneas \\ María José Rubio \\ Ruth Vilà \\ Universitat de Barcelona. España \\ aaneas@ub.edu \\ mjrubio@ub.edu \\ ruth_vila@ub.edu
}

Recibido: $26 / 2 / 2016$

Aceptado: 29/6/2016

Publicado: $26 / 10 / 2017$

\section{Resumen}

El objetivo de la investigación presentada ha sido conocer la percepción que tienen los alumnos que se encuentran en cuarto curso del grado de Pedagogía de la Universidad de Barcelona sobre sus competencias transversales antes y después de haber realizado la asignatura de Prácticas Externas con el uso de un portafolios digital. Mediante un diseño de pretest y postest, se ha desarrollado una investigación por encuesta con la cohorte de alumnos que empezaron el grado durante el curso 2011-2012 y que lo finalizaron durante el curso 2014-2015. El instrumento de recogida de datos ha sido el Cuestionario de apreciación de desempeño de competencias (12 ítems Likert-6 puntos según el nivel de aplicación de las competencias). Después de que los estudiantes hayan participado en las prácticas externas y de que hayan utilizado el portafolios digital en su evaluación, perciben que han conseguido un mayor nivel de autonomía en todas las competencias transversales. Se identifican dos perfiles de alumnado: un grupo que inicialmente tenía una percepción sobre su desarrollo competencial bastante bajo y que, en gran medida, mejora su percepción al finalizar la experiencia con portafolios digital, y otro grupo que tenía autopercepciones de un mayor desarrollo competencial al inicio y que, al finalizar, solo las mejora ligeramente.

Palabras clave: portafolios digital; evaluación; competencias transversales; prácticas externas; prácticum; Pedagogía

Resum. Portafolis digital $i$ avaluació de les competències transversals en les pràctiques externes del grau de Pedagogia de la Universitat de Barcelona

L'objectiu de la recerca presentada és valorar la percepció que tenen els alumnes que són a quart curs del grau de Pedagogia de la Universitat de Barcelona sobre les seves competències transversals abans i després d'haver realitzat l'assignatura de pràctiques externes amb l'ús d'un portafolis digital. Mitjançant un disseny de pretest i posttest, s'ha desenvolupat una recerca per enquesta amb la cohort d'alumnes que varen iniciar el grau durant el curs 2011-2012 i que el finalitzaren durant el curs 2014-2015. L'instrument de recollida de dades ha estat el Qüestionari d'apreciació d'acompliment de competències (12 ítems 
Likert-6 punts segons el nivell d'aplicació de les competències). Després que els estudiants hagin participat en les pràctiques externes i que hagin utilitzat el portafolis digital per avaluar-les, perceben que han assolit un nivell més alt d'autonomia en totes les competències transversals. S'identifiquen dos perfils d'alumnes: un grup que inicialment tenia una percepció sobre el seu desenvolupament competencial força baix i que, en gran mesura, millora la seva percepció en finalitzar l'experiència amb portafolis digital, i un altre grup que, al començament, tenia autopercepcions d'un desenvolupament competencial més elevat i que, en acabar, només les millora lleugerament.

Paraules clau: portafolis digital; avaluació; competències transversals; pràctiques externes; pràcticum; Pedagogia

Abstract. Digital portfolios and assessment of transversal competencies in internships of the Bachelor's Degree in Pedagogy at the University of Barcelona

This paper aims to assess the perception of $4^{\text {th }}$-year students enrolled in the Bachelor's Degree in Pedagogy at the University of Barcelona regarding transversal competencies before and after completing internships involving the use of a digital portfolio. Using a pretest and posttest design, a survey was administered to a cohort of students who entered the program in the 2011-2012 academic year and graduated in the 2014-2015 academic year. Data was collected by means of the Cuestionario de apreciación de desempeño de competencias (Competencies assessment questionnaire) comprising 12-items measured on a 6-point Likert scale according to the level of development of competencies. Following the internships and the use of digital portfolios for assessment purposes, students perceived a greater degree of autonomy in all transversal competencies. Two student profiles were identified: a group that initially perceived a relatively low competency development but whose perceptions significantly improved with the digital portfolios by the end of the internship and another group with higher self-perceptions of competency development at the beginning and which only slightly improved their perceptions upon completion of the internship.

Keywords: digital portfolios; assessment; transversal competencies; internship; practicum; pedagogy

\section{Sumario}

\section{Introducción 4. Método}

2. El prácticum en el grado de Pedagogía

5. Resultados
3. Objetivos y contexto
6. Conclusiones y discusión
de la investigación
Referencias bibliográficas

\section{Introducción}

La necesidad que tiene la universidad de orientarse hacia el desarrollo de competencias complejas demandadas por la sociedad y necesarias para los futuros profesionales ha llevado a las instituciones de educación superior a adoptar enfoques y modelos educativos basados en competencias (Bowden y Marton, 2012).

La importancia del prácticum como inmersión en una organización que permite desarrollar y aplicar competencias ha sido destacada extensamente (Zabalza, 1998; Ventura, 2005 o De Mora y Wood, 2014). Algunas de las 
más relevantes conclusiones de los estudios en esta línea destacan que los proyectos empresariales definidos y la inmersión en contextos profesionales que se desarrollan en el prácticum dan la oportunidad a los estudiantes para utilizar sus competencias en un contexto muy similar al que se encontrarán una vez graduados, lo cual facilitará su empleabilidad. Con una consistencia notable, los empresarios insisten en que, en el lugar de trabajo, los graduados deben ser capaces de ejecutar sus funciones, tener confianza, ser comunicadores, disponer de pensamiento crítico, resolver problemas y, además, ser personas adaptables. Estos requerimientos han permanecido imperturbables a pesar del momento actual de crisis, de transformación y de globalización. Informes como el de AC Nielsen Services Research (2000) o Coopers y Lybrand (1998) reconocen que una gran base de conocimientos disciplinarios no constituye, por sí misma, una garantía para asegurar la empleabilidad de los graduados. Por ejemplo, la investigación de Harvey (Harvey, Moon y Geall, 1997) realizada en el Reino Unido puso de relieve la importancia de que existiera una atribución interna y controlable, por parte del alumno, a la hora de valorar el éxito conseguido respecto a su rendimiento en el puesto de trabajo. Esta atribución se orienta a lograr que el estudiante sienta que dispone de las competencias necesarias y que es capaz de aplicarlas adecuadamente en el mundo laboral. En el mismo sentido, Harvey et al. (1997) y Te Wiata (2001) encontraron que la capacidad de los estudiantes estaba relacionada con el desarrollo de la confianza en su aplicación en contextos nuevos, diferentes y, sobre todo, profesionales, como sería el contexto del prácticum.

Por otro lado, son diversas las estrategias que pueden aplicarse en los procesos de evaluación por competencias. Ello depende de cómo se conceptualicen las competencias, tanto específicas como transversales, y la finalidad de dicha evaluación. En el caso de los procesos de evaluación por competencias transversales para el desarrollo de la empleabilidad, una de las estrategias más extensamente aplicadas es la del portafolios y, dentro de esta, destaca la modalidad del portafolios digital (De Miguel, 2005).

Numerosos autores y estudios coinciden en señalar que los portafolios digitales presentan importantes ventajas para los estudiantes relacionadas con el desarrollo de competencias transversales y metacognitivas, como la reflexión, el pensamiento crítico, la autonomía, el desarrollo profesional o la capacidad de organización y de autorregulación del aprendizaje (Galván, 2015; Rodrigues, 2013; Rubio y Galván, 2013; Sánchez Santamaría, 2012; Zubizarreta, 2009; Palomares, 2011; Cole, 2000; Corominas, 2000). Igualmente, se destaca la adecuación de los portafolios digitales en procesos de evaluación formativa (Klenowski, 2007; Lyons, 2003) y auténtica (Cambridge, 2010; Rodríguez Illera, 2009), siendo la evaluación auténtica aquella que permite a los estudiantes demostrar su aprendizaje a través de situaciones de la vida real y profesional (Meyer, 1992). El portafolios facilita la integración de la evaluación auténtica en el proceso de enseñanza-aprendizaje recopilando muestras de las actividades de aprendizaje y realizando una reflexión sobre los logros y las dificultades para la consecución de las competencias propuestas. En defi- 
nitiva, los portafolios desplazan su interés desde una evaluación del aprendizaje hasta una evaluación para el aprendizaje (Stiggins, 2008), que es aquella evaluación orientada a mejorar los procesos de aprendizaje, y no solo orientada a controlar y a certificar dichos procesos. El portafolios digital focaliza el centro de atención en el estudiante, lo que permite que este y el docente creen y gestionen un espacio virtual con las realizaciones personales, académicas y profesionales a través de acciones como escribir y archivar documentos, enlazar pensamientos (de manera explícita a través del hipertexto), contar la historia personal de aprendizaje a través de la evolución del portafolios y publicarla para que otras personas puedan leerla (Galván, 2015).

El discreto número de estudios sobre portafolios digitales en el contexto del prácticum (Ring y Foti, 2006; Raposo, Martínez y Tellado, 2009; Gallego, 2009; Morales, 2010; Cebrián, 2011; entre otros) destaca la importancia de estas herramientas en los procesos de aprendizaje y de seguimiento de las prácticas en organizaciones. La investigación de Cebrián (2011) evidenció el papel del portafolios digital para fomentar la comunicación dialógica entre estudiantes y docentes, siendo aquella una metodología que se muestra útil para los procesos de supervisión del prácticum. Por su parte, el estudio de Esqué, Gisbert y Larraz (2014) pone de relieve la ventaja del portafolios digital para los tutores que pueden, en la distancia, seguir el proceso del alumnado y reconducir situaciones no deseadas. Para el alumnado, el portafolios fomenta la capacidad de relacionar e integrar conocimientos y de reflexionar, aspectos que coinciden con el trabajo de otros autores (Palomares, 2007). El estudio de Morales (2010) en el prácticum de Educación Social demostró un alto grado de satisfacción del alumnado, acompañado de una gran autonomía, capacidad crítica y de toma de decisiones, todo mejorable con la inclusión de las TIC. Rico (2009) incide en la importancia del portafolios electrónico en la evaluación formativa y en la posibilidad de evidenciar el desarrollo de competencias a través de diferentes productos que los alumnos suelen desarrollar en las prácticas (fotos, vídeos, relatos, fichas, etc.). Además, los portafolios digitales presentan un valor añadido por su relevancia en los procesos de promoción académica y laboral y de empleabilidad de los graduados (Albuquerque y Cruz, 2008; Stefani, Mason y Pegler, 2007). El portafolios digital puede concebirse como un book profesional que puede ser incluido en redes y plataformas profesionales en donde puedan visibilizarse resultados y productos de aprendizaje acreditativos de cualificación. Zubizarreta (2009) incide en la idea de orientar los objetivos y la reflexión en el portafolios al desarrollo profesional.

\section{El prácticum en el grado de Pedagogía}

El prácticum del grado de Pedagogía de la Universidad de Barcelona se diseñó desde una lógica de ajuste a un perfil profesional contextualizado en base a los requerimientos y a las dinámicas de nuestra realidad social y profesional actual (Millan, Burguet, Vilà, Aneas, Rajadell y Noguera, 2014; Vilà, Burguet, Aneas, Rajadell, Noguera y Millan, 2014; Martínez, Rajadell, Aneas y Vilà, 
2016) y sobre una metodología que transitaba desde la exploración del perfil profesional (asignatura de Profesionalización y Salidas Laborales en primer curso) y la simulación (asignatura de Prácticas de Iniciación Profesional en tercer curso), hasta la inmersión en la realidad organizativa (asignatura de Prácticas Externas en el cuarto y último curso del título). Cabe aclarar que, si bien a lo largo del grado y de las dos primeras asignaturas del prácticum, el alumnado tiene la oportunidad de establecer contacto con diversas organizaciones y realidades profesionales, es en la asignatura de Prácticas Externas cuando este realmente se integra en una organización en la que puede aplicar y desarrollar las diversas competencias transversales y específicas de la asignatura, que son todas las del grado a excepción de las relacionadas con la investigación. Metodológicamente, esta asignatura tiene dos pilares: la inmersión en una organización en la que los estudiantes puedan ejercitar las competencias de la titulación desde el mayor nivel de autonomía posible y la participación en el Seminario de Práctica Reflexiva (Vilà y Aneas, 2013). Dicho seminario tiene las siguientes bases pedagógicas: $a$ ) la apreciación y la valorización del aprendizaje no formal tácito adquirido en el centro de trabajo (Eraut, 2000); b) el modelo de Práctica Reflexiva: R5 de Domingo (2009, 2010), que integra una secuencia de actividades individuales y colectivas de aprehensión, reconfiguración y expresión; c) las comunidades de práctica (Vásquez, 2011; Wenger, 2001), y d) el aprendizaje basado en problemas - ABP - (Bueno y Fitzgerald, 2004).

Por lo que respecta a la evaluación, los estándares europeos de garantía de calidad (Europe and Association for Quality Assurance in Higher Education, 2005) establecen que los estudiantes tendrían que estar claramente informados sobre los métodos de valoración a los que estarán sujetos, sobre qué se espera de ellos y sobre qué criterios se aplicarán para valorar su rendimiento. Todo esto implica que el reto que ahora tiene el profesorado de nuestras universidades consista en encontrar cómo desarrollar y cómo evaluar de manera formativa y coherente estos resultados de aprendizaje que se pretenden lograr a lo largo de los procesos de enseñanza-aprendizaje. Evaluar permite generar la evidencia de la adquisición de conocimientos, por tanto, a la vez que se describen los resultados obtenidos, es necesario determinar qué métodos y qué criterios de evaluación son los más adecuados para valorar si el estudiante ha adquirido el nivel de saberes, comprensión y competencias deseados. Los resultados del aprendizaje y los métodos de evaluación deben estar, por tanto, alineados. Y más allá de esta concepción de evaluación como «toma de decisiones» que nos es útil operativamente e indispensable al final del proceso de enseñanza-aprendizaje, la evaluación también es una parte consustancial del mismo proceso. Por lo tanto, las actividades de evaluación deben ser entendidas como una oportunidad estelar para generar aprendizajes, como un medio de mejora permanente, tanto desde el punto de vista de la adquisición de conocimientos como del proceso mismo. Así, analizar las estrategias de evaluación de los resultados de aprendizaje no solo supone focalizar la pertinencia y la coherencia del instrumento aplicado a tal efecto, sino también verificar si 
el desempeño del alumnado refleja el nivel de cualificación relativo al programa en que dicho alumnado se sitúa, en nuestro caso, el nivel 6 correspondiente al grado universitario (European Communities, 2008). En palabras de Sans (2008: 8): «La evaluación de los aprendizajes de los alumnos no es simplemente una actividad técnica o neutral, sino que constituye un elemento clave en la calidad del aprendizaje, ya que condicionará la profundidad y el nivel».

\section{Objetivos y contexto de la investigación}

El presente trabajo se plantea los siguientes objetivos:

- Conocer la percepción del alumnado de cuarto curso del grado de Pedagogía de la Universidad de Barcelona sobre sus competencias transversales, antes y después de haber realizado la asignatura de Prácticas Externas con el uso de un portafolios digital.

- Identificar posibles perfiles de alumnado respecto a esas percepciones sobre sus competencias transversales y el cambio al finalizar la asignatura.

La asignatura de Prácticas Externas supone un total de 18 ECTS (con 225 horas de inmersión en una organización de prácticas y 75 horas de trabajo tutelado en seminarios de práctica reflexiva [Vilà y Aneas, 2013] y 150 horas de trabajo autónomo). En la evaluación de los resultados de aprendizaje de esta asignatura, intervienen dos instrumentos con funciones muy diferenciadas en la misma: a) por un lado, el portafolio digital, utilizado como entorno en el que el alumnado irá integrando las diversas evidencias de su proceso de aprendizaje, y b) el CADC - Cuestionario de Apreciación de Desempeño de Competencias- (Vilà y Aneas, 2013). Este instrumento, cumplimentado por el alumnado, el tutor de universidad y el tutor de la organización de prácticas, tiene una finalidad claramente formativa, en cuanto a que su cumplimentación requiere una profunda reflexión, por parte de quien lo contesta, sobre los diversos logros y aprendizajes alcanzados por el alumnado en sus prácticas externas. Este modelo de evaluación ha aportado evidencias de utilidad y del grado de desempeño aplicado por el alumnado desde el punto de vista de los tres agentes que intervienen en la evaluación de la asignatura (Aneas, Vilà y Alós, 2015; Aneas, Reguant y Rodríguez, 2015; Aneas y Vilà, 2015; Vilà, Aneas y Rajadell, 2015; Aneas, Vilà, Armadans y Cid, 2013).

El portafolios digital es introducido en las prácticas externas como sistema de evaluación alternativa, formativa y auténtica. Para el alumnado, el portafolios tiene el objetivo de representar su carpeta de aprendizajes realizados en las prácticas, una forma de evidenciar el desarrollo de sus competencias. Las competencias específicas y transversales de la asignatura están publicadas en el plan docente y se explicitan en los seminarios de práctica reflexiva.

Para asegurar un uso eficaz en el proceso de implementación del portafolios digital, se siguió un tratamiento didáctico adecuado a los objetivos, aspecto relevante en las metodologías centradas en el estudiante y su desempeño y su 
autonomía personal (Biggs, Kember y Leung, 2001). Este tratamiento didáctico fue consensuado por el equipo docente y se basó en tres aspectos considerados esenciales en relación con los portafolios digitales (Delandshere y Arens, 2003; Barberà, 2005; Rubio, Galván y Rodríguez, 2013):

1. Formación al alumnado y al profesorado sobre el conocimiento de lo que es un portafolios digital y del sistema de portafolios utilizado. Esta formación fue complementada con el soporte técnico a lo largo del curso en aspectos relacionados con la plataforma.

2. Realización de un portafolios individual de manera continuada, periódica y formativa. El alumnado dispuso de todo un curso académico para realizar el portafolios, un tiempo mínimamente considerado para conseguir el empoderamiento con el portafolios digital. Su realización fue progresiva con diferentes periodos de publicación.

$\mathrm{Al}$ alumnado se le orienta sobre el diseño y la organización del portafolios, instándole a crear uno con una navegación clara, intuitiva y fluida, que contenga una serie de secciones (preestablecidas y para todos igual) para ordenar el contenido, unas evidencias de aprendizaje dentro de cada sección y un hilo conductor sobre las mismas; esto último en lo que Barberà (2008) denomina "trama del portafolios", que puede ser implícita o esquemática o explícita y explicativa, donde prima la narración de una historia a la que secundan las evidencias. Se solicitaron evidencias de aprendizaje orientadas al autoconocimiento para el desarrollo personal y profesional, tal y como se aprecia en la tabla 1.

3. Retroalimentación activa del docente sobre las diferentes versiones del portafolios que publican los estudiantes. En la experiencia presentada, el profesorado ha asumido la labor de guía y facilitador del aprendizaje, orientando dicho proceso e implicándose activamente en la tarea de evaluación (Klenowski, 2007). El docente tiene un papel importante en el desarrollo de competencias del alumnado, por lo que la acción de retroalimentar a los estudiantes debe ser intrínseca al uso de portafolios digitales. En el portafolios digital, la retroacción es permanente, por la posibilidad que tiene el docente de acceder continuamente al portafolios y la que tiene el estudiante de actualizarlo de manera continua.

La plataforma utilizada (Carpeta Digital, diseño del grupo GREAV de la Facultad de Educación de la Universidad de Barcelona) facilita los procesos de autorregulación del aprendizaje (Zimmerman, 2002) por las diversas funcionalidades que posee, como la comunicación entre docente y estudiante, la publicación del portafolios para ser visionado y comentado por otras personas (entre ellas, el docente), la posibilidad de que el estudiante establezca una relación entre evidencias y competencias o la posibilidad de organizar el portafolios a través de secciones. 
Tabla 1. Secciones del portafolios

\begin{abstract}
1. Análisis de la organización
Esta actividad supone la descripción y el análisis de la institución de prácticas aplicando los contenidos (organización, recursos y servicios, atención a usuario, trabajo en equipo, comunicación, diversidad, calidad, sostenibilidad e innovación) y las reflexiones de las sesiones de práctica reflexiva.
\end{abstract}

\title{
2. Proyecto de implicación
}

Esta actividad supone la realización de un proyecto cuya destinataria final es la institución de prácticas. Planteado como un output o un producto profesional que puede tener una naturaleza y un formato diversos (estudio de necesidades, desarrollo de un programa, diseño y realización de un recurso didáctico, etc.).

3. Proyecto de desarrollo e inserción profesional

Esta actividad supone un trabajo donde se formulará el objetivo profesional, el plan de búsqueda de empleo y el plan de formación continua.

\section{Memoria de aprendizajes}

Este componente del portafolios es una memoria que aporta evidencias del proceso de autoconocimiento y de autoevaluación sobre las competencias alcanzadas, las responsabilidades desarrolladas y los aprendizajes generados en las SPR y a lo largo de la estancia en la institución.

5. Currículum vítae

Fuente: Aneas, A y Vilà R. (2012)

\section{Método}

Las preguntas que guían este estudio plantean si el uso del portafolios digital puede ayudar a mejorar la percepción de desarrollo competencial en el alumnado y si existen diferentes perfiles en cuanto al desarrollo competencial alcanzado. Para dar respuesta a dichas preguntas, se ha desarrollado una investigación por encuesta con la cohorte de alumnos que empezaron el grado de Pedagogía durante el curso 2011-2012 y que lo finalizaron durante el curso 2014-2015, en un diseño de pretest y postest.

El instrumento utilizado en la recogida de datos ha sido el CADC - Cuestionario de apreciación de desempeño de competencias - (Vilà y Aneas, 2013), cumplimentado únicamente por el alumnado, sobre sus diversos logros y aprendizajes en las prácticas externas, únicamente en las competencias transversales. La escala de competencias transversales consta de 12 ítems Likert de 6 puntos según el nivel de aplicación de las competencias. Se ha apostado por las categorías de los instrumentos en el Marco Europeo de Cualificaciones Profesionales (EQF-MEC), de las Comunidades Europeas (2008). Los indicadores de competencias se basan fundamentalmente en términos de autonomía y en la capacidad de transferir. El CADC, además de aportar indicadores sobre si se había apreciado la aplicación de una competencia, pretende aportar orientación sobre el nivel de cualificación de dicho desempeño. Así, la escala sobre competencias transversales tiene seis niveles de desarrollo. Sucesivas revisiones por expertos, usuarios y aplicaciones piloto permitieron realizar su validación definitiva en cuanto al contenido y a la comprensibilidad (Vilà y Aneas, 2013). 
Respecto a su dimensión psicométrica, cabe mencionar que la escala ha obtenido coeficientes alfa de Cronbach de fiabilidad, de 0,991 (pretest) y 0,833 (postest). Así pues, el cuestionario cuenta con el rigor científico necesario para ser utilizado.

Los participantes han sido 203 alumnos que, de forma voluntaria, han cumplimentado el cuestionario en la modalidad en línea (CADC) con ítems escalares respecto a su apreciación sobre el nivel de autonomía en que se habían aplicado las competencias transversales del grado de Pedagogía, en dos momentos distintos a lo largo del grado (tercer y cuarto cursos). Considerando que la población de todo el alumnado de último curso del grado de Pedagogía es aproximadamente de 225 alumnos, se dispone de un índice de respuesta del $90 \%$, que supone un error de 0,022.

Siendo en dos momentos distintos, la muestra del primer momento consta de 169 alumnos, y en el segundo momento, de 202. Hay 168 alumnos que han coincidido en ambas aplicaciones. Considerando la población, se dispone de un índice de respuesta en la muestra total (con sujetos coincidentes en ambas aplicaciones) del $75 \%$, que supone un error de 0,039 .

El análisis de los resultados se ha llevado a cabo mediante estadística descriptiva, aplicando contrastes de medias para muestras relacionadas ( $t$ de Student) y análisis de la fiabilidad de la escala con Alpha de Crombach. Se ha aplicado también el procedimiento de análisis de conglomerados en dos fases (bietápico), el cual permite seleccionar automáticamente el número óptimo de conglomerados (Bacher, Wenzig y Vogler, 2004). Todos estos cálculos se han realizado con el paquete estadístico SPSS, versión 18.

\section{Resultados}

\subsection{Las competencias transversales en tercer curso del grado de Pedagogía}

En general, el alumnado de tercer curso percibe que dispone de un nivel medio-bajo en cuanto a las competencias transversales. Las medias que se obtienen del grupo oscilan entre 2,3 puntos (sobre 6) y 3,2 (sobre 6).

Tal como se resume en la tabla 2 , mayoritariamente, dichas competencias transversales no superan la media teórica posible de la escala (3 puntos), cosa que indica un nivel limitado de autonomía en la competencia por parte de este alumnado. Destacan especialmente las competencias de sostenibilidad, actitud innovadora, resolución de problemas y adaptación al cambio como las que se perciben como menos desarrolladas por parte del alumnado en este tercer curso. Por otra parte, las competencias que se perciben con mayor autonomía por parte de los participantes en este primer momento de recogida de datos son: aprender y ser responsable, compromiso ético y autoconocimiento. No obstante, las puntuaciones obtenidas en estas competencias transversales oscilan entre 2,9 y 3,2 (sobre 6), por tanto, tampoco esta percepción es destacadamente alta. 
Tabla 2. Puntuaciones medias obtenidas en el pretest y en el postest en las competencias transversales y en los estadísticos de contraste

\begin{tabular}{|c|c|c|c|c|c|}
\hline & $\begin{array}{l}\text { Media } \\
\text { pretest }\end{array}$ & $\begin{array}{l}\text { Media } \\
\text { postest }\end{array}$ & $\boldsymbol{t}$ & $\begin{array}{l}\text { GI } \\
\text { Grados } \\
\text { de } \\
\text { libertad }\end{array}$ & $\begin{array}{l}\text { P } \\
\text { Grado } \\
\text { de signi- } \\
\text { ficación }\end{array}$ \\
\hline 1. Compromiso ético & 2,8795 & 4,7229 & $-9,371$ & 165 & 0,000 \\
\hline 2. Aprender y ser responsable & 3,2143 & 5,2798 & $-9,961$ & 167 & 0,000 \\
\hline 3. Trabajar en equipo & 2,8036 & 5,4643 & $-12,478$ & 167 & 0,000 \\
\hline 4. Creatividad e iniciativa & 2,5269 & 4,5629 & $-10,432$ & 166 & 0,000 \\
\hline 5. Sostenibilidad & 2,2683 & 4,1037 & $-10,387$ & 163 & 0,000 \\
\hline 6. Comunicar & 2,7470 & 4,7229 & $-9,577$ & 165 & 0,000 \\
\hline 7. Aplicar el conocimiento pedagógico & 2,6548 & 4,6429 & $-9,872$ & 167 & 0,000 \\
\hline $\begin{array}{l}\text { 8. Autoconocimiento para el desarrollo } \\
\text { personal y profesional }\end{array}$ & 2,8512 & 5,1607 & $-11,040$ & 167 & 0,000 \\
\hline 9. Conciencia compleja & 2,6548 & 4,7798 & $-10,962$ & 167 & 0,000 \\
\hline $\begin{array}{l}\text { 10. Resolución de problemas educativos } \\
\text { desde una perspectiva multicultural } \\
\text { y compleja }\end{array}$ & 2,4762 & 4,5417 & $-11,130$ & 167 & 0,000 \\
\hline 11. Actitud innovadora & 2,4671 & 4,3952 & $-10,706$ & 166 & 0,000 \\
\hline $\begin{array}{l}\text { 12. Adaptación al cambio en la sociedad } \\
\text { del conocimiento }\end{array}$ & 2,4970 & 4,6909 & $-11,460$ & 164 & 0,000 \\
\hline
\end{tabular}

Fuente: elaboración propia.

\subsection{Las competencias transversales después de participar en la evaluación con portafolios digital}

En la aplicación del CADC en el curso siguiente (último del grado de Pedagogía) y después de que el alumnado participara en las prácticas externas y se usara el portafolios digital en su evaluación, los estudiantes perciben tener un mayor nivel de autonomía en las competencias transversales (ver tabla 2). En esta segunda ocasión, las puntuaciones medias obtenidas en las competencias por parte del grupo de participantes oscilan entre 4,1 y 5,5 (sobre 6). En esta segunda aplicación, se perciben con un nivel notable la autonomía en estas competencias. Destaca nuevamente la sostenibilidad como la competencia con menor puntuación. Y destacan también las competencias trabajar en equipo; aprender y ser responsable, y autoconocimiento como las que tienen mayores puntuaciones en esta segunda aplicación.

De hecho, tal como se resume en la tabla 2, todas las competencias aumentan de forma estadísticamente significativa en la segunda aplicación respecto a la primera. Es decir que, en todos los casos, las percepciones del alumnado son de mayor grado de autonomía al finalizar el grado de Pedagogía respecto a lo que percibían en el curso anterior. Este aumento se representa en el gráfico 1.

A pesar de que todos los cambios han sido estadísticamente significativos, destacan algunas competencias cuyo aumento al finalizar el grado es más espectacular, como, por ejemplo: trabajar en equipo; adaptación al cambio; resolución 
Gráfico 1. Medias obtenidas en el pretest y en el postest en las competencias transversales percibidas

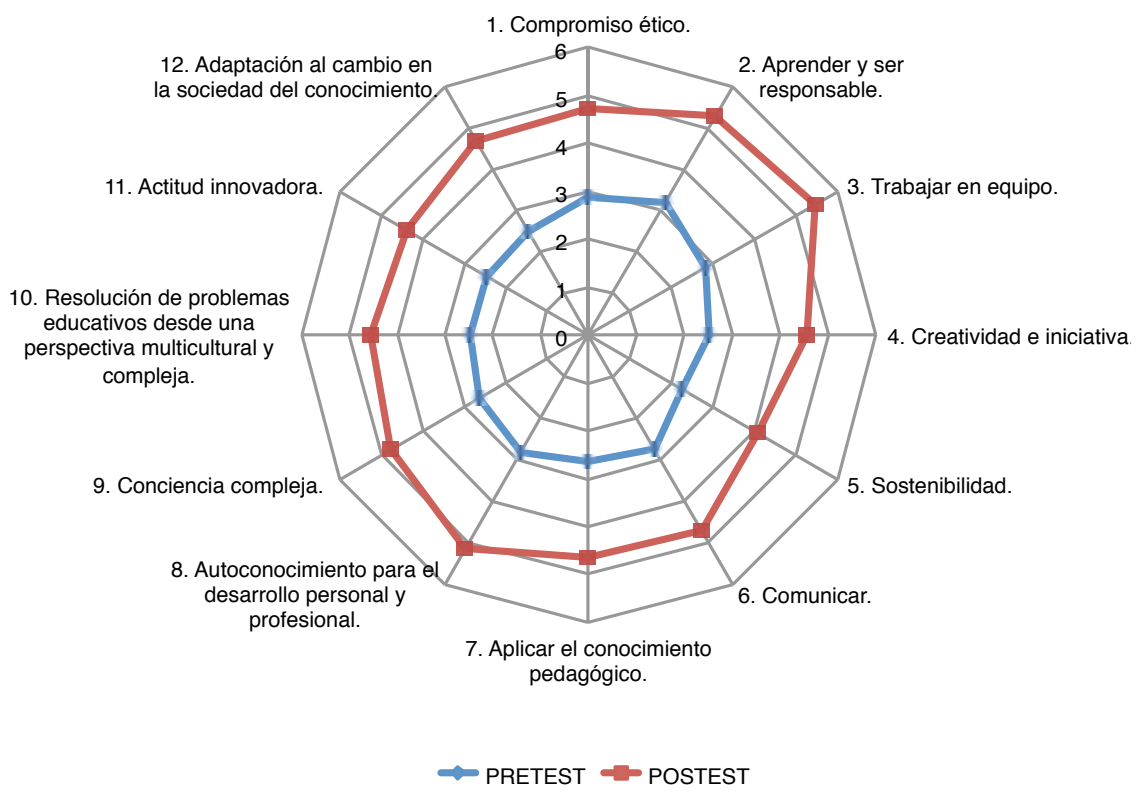

Fuente: elaboración propia.

de problemas educativos desde una perspectiva multicultural y compleja; autoconocimiento para el desarrollo personal y profesional, y conciencia compleja.

\subsection{Perfiles de alumnado sobre la percepción de su desarrollo competencial}

Considerando la media de desarrollo competencial percibido en la aplicación inicial en tercer curso del grado de Pedagogía (DC1) y la media del desarrollo competencial que percibe el alumnado al finalizar el grado de Pedagogía (DC2), así como el cambio que supone el diferencial entre ambas medias (DC2-DC1), se identifican dos perfiles de alumnado en un modelo significativo, considerando la calidad de los conglomerados (figura 1). 
Figura 1. Resumen del modelo bietápico

Resumen del modelo

\begin{tabular}{|ll|}
\hline Algoritmo & Dos fases \\
\hline Características de entrada & 3 \\
\hline Agrupaciones & 2 \\
\hline
\end{tabular}

Calidad de conglomerados

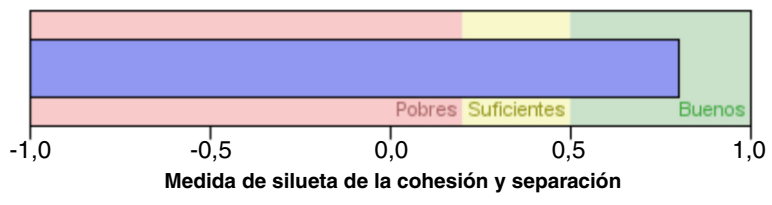

Fuente: elaboración propia. ra 2):

Los dos perfiles identificados corresponden a los siguientes alumnos (figu-

- Perfil 1. Grupo de mejora en la percepción sobre sus competencias transversales después de la experiencia con portafolios digital. Este grupo corresponde al $44 \%$ del alumnado participante y está formado por aquellos estudiantes que inicialmente tenían una percepción sobre su desarrollo competencial bastante bajo en cuanto a las competencias transversales. En cambio, al finalizar la experiencia con portafolios digital en la asignatura de Prácticas Externas, su percepción mejora en gran medida. Por tanto, muestran un cambio muy superior de aumento en las puntuaciones respecto al cambio que presenta el otro grupo.

- Perfil 2. Grupo estable en su percepción sobre sus competencias transversales antes y después de la experiencia con portafolios digital. Este grupo corresponde al 56\% del alumnado participante. Se trata de aquellos estudiantes que, en el tercer curso del grado, ya tenían autopercepciones de un mayor desarrollo competencial a nivel de las asignaturas transversales. Al finalizar el grado de Pedagogía, sus percepciones mejoran solo ligeramente.

\section{Conclusiones y discusión}

En respuesta al primer objetivo del estudio, la conclusión principal refuerza la idea de la importancia del prácticum y del portafolios digital en el desarrollo de competencias. Tras la realización de las prácticas externas y el uso del portafolios digital en la evaluación, el alumnado percibe tener un mayor nivel de 
Figura 2. Perfiles de alumnado según las tres variables introducidas en el modelo

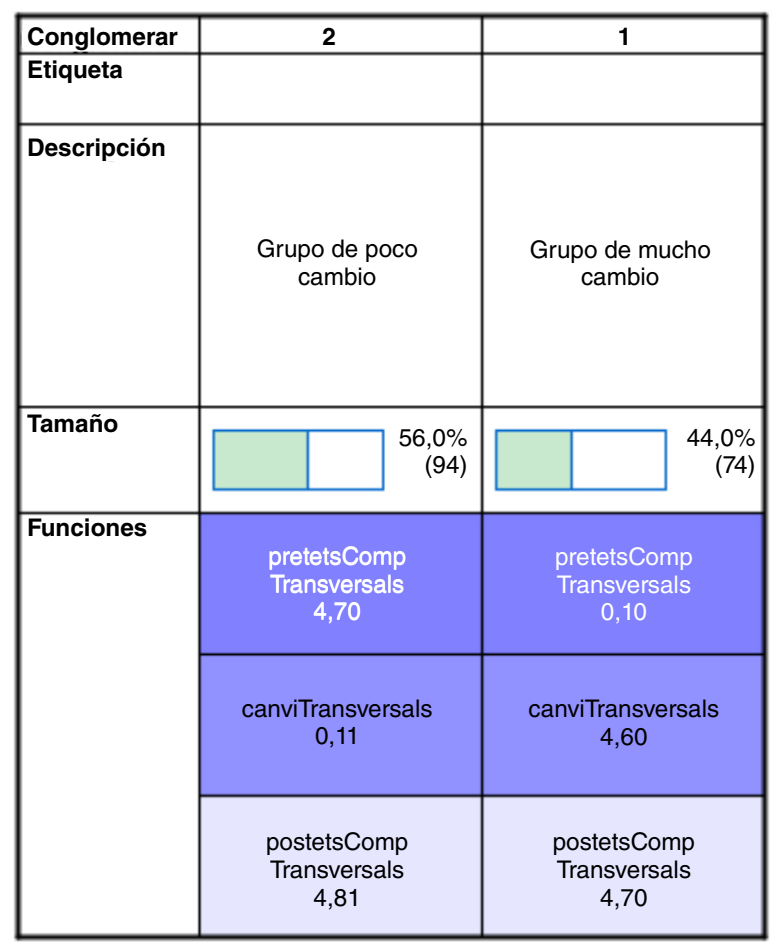

Fuente: elaboración propia.

autonomía en las competencias transversales propuestas en el plan docente. La interpretación de dicha mejora la podemos encontrar en las propias teorías que subyacen al prácticum y a los portafolios, aunque debería confirmarse en futuros estudios.

Por un lado, la propia literatura ya destaca que el desarrollo efectivo de las competencias depende de las oportunidades para ponerlas en práctica en un contexto (Drummond, Nixon y Wiltshire, 1998; Zabalza, 1998; entre otros), de tal manera que, efectivamente, podría interpretarse que el alumnado dispone de un buen potencial de competencia (adquirida a lo largo del grado y de otras experiencias formativas y ocupacionales) y que el contacto con la organización de prácticas, gracias al modelo inmersivo, ha sido el catalizador que ha permitido su desempeño.

En efecto, el prácticum contribuye a desarrollar un conjunto de competencias inherentes al carácter regulativo y práctico de la profesión, como son: la ética profesional, la sostenibilidad, el trabajo en equipo o la aplicación del conocimiento pedagógico desde una perspectiva multicultural y compleja y la 
actitud innovadora. En esta línea, el estudio de Gómez, Sánchez y Núñez (2011) evidenció que los estudiantes consideraron que las prácticas habían mejorado sus habilidades de organización y planificación, toma de decisiones y trabajo en equipos multiprofesionales, y que habían ampliado su apreciación de la diversidad y la multiculturalidad que existe en los contextos profesionales del psicopedagogo. En la misma línea, los resultados se ajustan a los trabajos de Harvey et al. (1997) y de Te Wiata (2001) citados en la introducción del presente artículo.

Por otro lado, se constata la importancia de los portafolios digitales en los procesos de autorregulación del aprendizaje, de vital importancia para el desarrollo de la autonomía (Zimmerman, 2002; Wolters, 2003; Rosário, Lourenço, Paiva, Núñez y González-Pienda, 2012). Dichos procesos permiten al estudiante saber cuál es su objetivo de aprendizaje, planificar su trabajo para conseguirlo, aplicar conscientemente estrategias y evaluar constantemente tanto el proceso en sí mismo como el resultado de sus prácticas. Por ello, el portafolios puede contribuir a facilitar el autoconocimiento para el desarrollo personal y profesional, también contribuye a conseguir autonomía, a aprender a aprender o a la capacidad de realizar comunicación escrita, como han evidenciado también otros autores (Morales, 2010). En estas competencias, intervienen procesos de metacognición y autorregulación, como son la planificación, el desempeño y la mejora, además de la autoevaluación y la autorreflexión, aspectos considerados presentes en el proceso de construcción de portafolios digitales (Plaisir, Hachey y Theilheimer, 2011; Hallam y Creagh, 2010). En la investigación cualitativa de Galván (2015), llevada a cabo con diferentes estudiantes procedentes de diversas disciplinas, se constató que los estudiantes piensan metacognitivamente a la hora de diseñar el portafolios digital (codifican el proceso de aprendizaje de elaboración del portafolios digital, hacen ajustes de mejora en las actividades y en los portafolios siguientes y transfieren el proceso a nuevas situaciones y también a otros portafolios). La capacidad de comunicación escrita gracias a los portafolios digitales también se ha puesto de manifiesto en varios estudios (Loureiro, Moreira y Gomes, 2008; Sánchez Santamaría, 2012).

Con relación al segundo objetivo, los resultados han puesto de manifiesto la presencia de dos perfiles de alumnado: aquel que mejora su percepción sobre las competencias transversales después de la experiencia con portafolios digital, y aquel que es más estable en sus percepciones sobre las competencias transversales antes y después de la experiencia. Ante la existencia de esos dos tipos de alumnado, se abren diversos interrogantes según la mejora de su percepción. Dichos interrogantes deberán ser indagados en futuros estudios, en el sentido de identificar qué tipo de variables (experiencias profesionales previas, rasgos de personalidad, antecedentes de éxito académico en la carrera, etc.) pueden motivar esas diferencias.

Los resultados del presente estudio adquieren un significado muy relevante si se relacionan con los postulados sobre la atribución y la percepción de competencia de Harvey (1999). Así, como conclusión final, deberíamos con- 
siderar el valor que tienen las estrategias docentes e institucionales combinadas y propuestas en este estudio para el aprendizaje. Concretamente, apostar por un modelo de prácticas basado en el aprendizaje en contextos reales como son las organizaciones empresariales está demostrando efectos positivos en el fomento de un conjunto de competencias profesionalizadoras, al mismo tiempo que el portafolios digital fomenta competencias de tipo metacognitivo necesarias asimismo para el contexto profesional. Otros estudios también han constatado la importancia de esta combinación (Esteve-González et al., 2015, y los citados en la introducción de este artículo). Tanto el diseño como la estrategia evaluativa de la asignatura de Prácticas Externas del grado de Pedagogía de la Universidad de Barcelona han podido ejercer una influencia positiva en la mejora de la empleabilidad de los graduados, hipótesis que deberá verificarse en futuros estudios sobre la inserción laboral de los mismos. Igualmente, futuras investigaciones deberán permitir detallar cuál ha sido el peso específico del portafolios digital en la mejora de la percepción de la propia competencia de los graduados.

\section{Referencias bibliográficas}

AC Nielsen Research Services (2000). Employer Satisfaction with Graduate Skills: Research Report. Evaluations and Investigations Programme, Higher Education Division. Canberra: Department of Education, Training and Youth Affairs.

Albuquerque, F. y Cruz, E. (2008). Analysing ICT tools for Portfolio Educational. En: Albuquerque, F. y Laranjeiro, M. (eds.). E-portfolio in Education: Practices and Reflections (pp. 75-82). Mem Martins: Associaçao de Professores de Sintra.

Aneas, A.; Reguant, M. y Rodríguez, M.L. (2015). Evaluación de los resultados de aprendizaje: El caso del prácticum del grado de Pedagogía de la Universidad de Barcelona. En AIDIPE (ed.). Investigar con y para la sociedad, 2 (pp. 1061-1071). Cádiz.

Aneas, A. y Vilà, R. (2012). Pràctiques externes Grau de Pedagogia: Guia per l'alumnat. Universitat de Barcelona. Dipòsit Digital. <http://hdl.handle.net/2445/33740>

- (2015). ¿Hay cambios en la percepción que tiene el alumnado sobre su competencia tras haber desarrollado su prácticum?: El caso del prácticum del grado de Pedagogía de la Universidad de Barcelona. En AIDIPE (ed.). Investigar con y para la sociedad, 2 (pp. 1073-1084). Cádiz.

Aneas, A.; Vilà, R. y Alós, M. (2015). La vinculació de les pràctiques externes i el treball final de grau, percepcions de l'alumnat sobre el seu desenvolupament competencial. En UNIVEST (ed.). Los retos de mejorar la evaluación (pp. 402-408). Dipòsit Digital de la Universitat de Girona. <http://dugi-doc.udg.edu/handle/ $10256 / 10804>$

Aneas, A.; Vilà, R.; Armadans, I. y Cid, A. (2013). Evaluación de las competencias transversales del grado de Pedagogía de la Universidad de Barcelona. Academia Journals, 5(3), 23-35.

Armadans, I.; Castrechini, A. y Aneas, A. (2013). Empleabilidad, prácticas externas y autorregulación del aprendizaje en la universidad: Propuestas y recomendaciones desde una perspectiva transdisciplinar. En Muñoz, P.C.; Raposo, M.; González, M.; Martínez, M.E.; Zabalza, M.A. y Pérez, A. (coord.). Alejando 
las paralelas y acrecentando las perpendiculares (pp. 159-167). Santiago de Compostela: Andavira.

Armengol, J.; Hernández, J. y Mora, J. (2009). Experiencias sobre el uso del portafolio del estudiante en la UPC. Revista de Educación a Distancia. Monográfico VIII, 1-17.

Bacher, J.; Wenzig, K. y Vogler, M. (2004). SPSS Two Step Cluster, a First Evaluation. Arbeits-Und, Diskussionpapiere, 2(2), 23-34.

BARBERÀ, E. (2005). La evaluación de competencias complejas: La práctica del portafolio. La Revista Venezolana de Educación (Educere), 9(31), 497-503.

- (2008). El estilo E-portafolio. Barcelona: UOC.

BARRAGÁN, R. (2005). El portafolio, metodología de evaluación y aprendizaje de cara al nuevo EEES: Una experiencia práctica en la universidad de Sevilla. Revista Latinoamericana de Tecnología Educativa, 4(1), 121-139.

Biggs, J.; Kember, D. y Leung, D. (2001). The revised two-factor study process qüestionari: R-SPQ-2F. British Journal of Educational Pshychology, 71, 133-149.

Bowden, J. y MARTON, F. (2012). La Universidad: Un espacio para el aprendizaje más allà de la calidad y la competencia. Madrid: Narcea.

Bueno, P.M. y FitzGerald, V.L. (2004). Aprendizaje basado en problemas. Theoria, $13,145-157$.

Cambridge, D. (2010). Eportfolios for Lifelong Learning and Assessment. San Francisco: Jossey-Bass.

Cebrián, M. (2011). Supervisión con e-portafolios y su impacto en las reflexiones de los estudiantes en el Practicum: Estudio de caso. Revista de Educación, 354, 183208.

Cole, D.J. (2000). Porffolios Across the Curriculum and Beyond. California: Sage Publications.

Coopers, J. y Lybrand, M. (1998). Skills Development in Higher Education. Londres: Coopers \& Lybrand.

Corominas, E. (2000). ¿Entramos en la era del portafolios? Bordón, 52(4), 509-521.

Delandshere, G. y Arens, S.A. (2003). Examining the quality of the evidence inpreservice teacher portfolios. Journal of Teacher Education, 54, 57-73.

De Mora, J. C. y Wood, K. (2014). Practical knowledge in teacher education: Approaches to teacher internship programmes. Routledge.

Domingo, A. (2009). El Prácticum en los nuevos planes de estudios. En M. Raposo, E. Martínez y L. Lodeiro (coord.). Formación vs Training (pp. 125-131). Santiago de Compostela: Imprenta Universitaria.

- (2010). La práctica reflexiva en los estudios de Magisterio de la UIC. En O. EsteVE (coord.). Creando mi profesión: Una propuesta para el desarrollo profesional del profesorado (pp. 97-114). Barcelona: Octaedro.

Drummond, I.; Nixon, I. y Wiltshire, J. (1998). Personal transferable skills in higher education: The problems of implementing good practice. Quality Assurance in Education, 6(1), 19-27.

Eraut, M. (2000). Non-formal learning and tacit knowledge in professional work. British. Journal of Educational Psychology, 70, 113-136.

Esqué, S.; Gisbert, M. y LarRaz, V. (2014). El uso del e-portafolios en las prácticas del Bàtxelor en Enfermería. REDU: Revista de Docencia Universitaria, 12(1), 399-423.

Esteve-González, V.; Sánchez, A.; Marqués, L.; Holgado, J. y Cela, J.M. (2015). Diseño de un portafolio digital para la validación de competencias de los 
estudiantes universitarios de las prácticas externas en las escuelas. Congreso EDUTEC. Ecuador.

Europe and Association for Quality Assurance in Higher Education (2005). Criterios y Directrices para la garantía de Calidad en el Espacio Europeo de Educación Superior. Recuperado de <http://www.enqa.eu/indirme/esg/ESG\%20in\%20Spanish_by\%20ANECA.pdf>.

European Communities (2008). European Qualification Framework for the live-long Learning. Recuperado de <https://ec.europa.eu/ploteus/sites/eac-eqf/files/leaflet_ en.pdf>.

Gallego, M.J.; Gámiz, V.; Pérez, P. y Romero, A. (2009). Desarrollo de competencias en el Prácticum con materiales y actividades online. Pixel-Bit: Revista de Medios y Educación, 34, 135-150.

Galván, C. (2015). El desarrollo del conocimiento metacognitivo en los portafolios digitales de Educación Superior. Tesis doctoral presentada en la Universidad de Barcelona.

GibBs, G. (2003). Uso estratégico de la evaluación en el aprendizaje. En S. Brown y A. Glasner (eds.). Evaluar en la universidad: Problemas y nuevos enfoques (pp. 61-74). Madrid: Narcea.

Gómez, P.; Sánchez, R. y NúÑez, C. (2011). La perspectiva de los estudiantes sobre la adquisicion de competencias en las prácticas externas: Experiencias innovadoras en el aprendizaje a través de la evaluación. Revista de Docencia Universitaria, 9(2), 256-265.

Hallam, G. y Creagh, T. (2010). ePortfolio use by university students in Australia: A review of the Australian ePortfolio Project. Higher Education Research \& Development, 5, 179-193.

Harvey, L. (1999). New Realities: The Relationship between Higher Education and Employment. Keynote presentation at the European Association of Institutional Research Forum, Lund, Sweden, August. <http://www.qualityresearchinternational.com/Harvey\%20papers/Harvey\%201999\%20New\%20Realities\%20 EAIR\%20Lund.pdf>. Accedido el 3 de julio 2013

Harvey, L.; Moon, S. y Geall, V. (1997). Graduates' Work: Organisational Change and Students' Attributes. Birmingham: CRQ, AGR.

Klenowski, V. (2007). Desarrollo de portafolios: Para el aprendizaje y la evaluación. Madrid: Narcea.

Loureiro, M.J.; Moreira, A. y Gomes, M.J. (2008). ePortfolios and eArgumentation. En F. Albuquerque y M. Laranjeiro (eds.). e-portfolio in Education: Practices and Reflections (pp. 53-60). Mem Martins: Associaçao de Professores de Sintra.

Lyons, N. (ed.) (2003). El uso del portafolios. Buenos Aires: Amorrortu.

Martínez, S.; Rajadell, N.; Aneas, A. y Vilà, R. (2016). Repensar la profesión desde la formación y la práctica: Las Prácticas Externas de Pedagogía. RIDURevista d'Innovació Docent i Universitària, 8(16), 98-108. <http://dx.doi.org/10.1344/RIDU2016.8.11>

Mendoza, R. de; Domínguez, R. y Martín, E. (2002). Metodología docente utilizada por el profesorado universitario. Cultura y Educación, 14 (2), 177-186.

Meyer, C. (1992). What's the difference between authentic and performance assessment? Education Leadership, 49(8), 39-40.

Miguel, M. de (coord.) (2005). Modalidades de enseñanza en el desarrollo de competencias: Orientaciones para promover el cambio metodológico en el marco del EEES. Oviedo: Ediciones de la Universidad de Oviedo. 
Millan, D.; Burguet, M.; Vilà, R.; Aneas, A.; Rajadell, N. y Noguera, E. (2014). PRAXIS: El pràcticum al grau de Pedagogia de la Universitat de Barcelona. Revista d'Innovació Docent Universitària, 6, 32-52.

Morales, S. (2010). El Practicum en educación social: El portafolio como instrumento de seguimiento y evaluación del aprendizaje de los alumnos. Revista Docencia e Investigación, 20, 33-58.

Palincsar, A.S. (1998). Social constructivist perspectives on teaching and learning. Annual Review of Psichology, 49, 345-375.

Palomares, A (2007). El portafolios como metodología activa en el diseño y desarrolo del Practicum. En IX Symposium Internacional sobre Practicum y Prácticas en empresas en la formación universitaria. Poio (Pontevedra): Universidad de Vigo.

- (2011). El modelo docente universitario y el uso de nuevas metodologías en la enseñanza, aprendizaje y evaluación. Revista de Educación, 355 (mayo-agosto), 591-604.

Plaisir, J.Y.; Hachey, A.C. y Theilheimer, R. (2011). Their portfolios, our rol: Examining a community college teacher education digital portfolio program from the students perspective. Journal of Early Chilhood Teacher Education, 32(2), 159-175.

Raposo, M.; Martínez, M. y Tellado, F. (2009). El e-portafolios como recurso para el Practicum en el grado de educación social. X Symposium Internacional sobre Practicum. Poio (Pontevedra): Universidad de Vigo.

Rico, A.M. (2009). El portafolios en las prácticas de enseñanza del grado en Maestro en Educación Primaria. REIFOP, 13(3), 37-45.

Ring, G. y Foti, S. (2006). Using ePortfolios to Facilitate Professional Development Among Pre-Service Teachers. En A. Jafari y C. Kaufman (eds.). Handbook of research on ePortfolios (pp. 340-357). Hershey, PA, USA: IGI Publishing.

Rodrigues, R. (2013). El desarrollo de la práctica reflexiva sobre el quehacer docente, apoyada en el uso de un portafolio digital, en el marco de un programa de formación para académicos de la Universidad Centroamericana de Nicaragua. Tesis doctoral presentada en la Universidad de Barcelona.

Rodríguez Illera, J.L. (2009). Los portafolios digitales como herramientas de evaluación y de planificación personal. En M. CASTELló (comp). La evaluación auténtica en Enseñanza Secundaria y universitaria: Investigación e innovación (pp. 145163). Barcelona: Edebé.

Rodríguez Moreno, M.L.; Serreria, P. y Cimmuto, P. del (2010). Desarrollo de competencias: Teoría y práctica. Barcelona: Laertes.

Rosário, P.; Lourenço, A.; Paiva, M.O.; Núñez, J.C. y González-Pienda, J.A. (2012). Autoeficacia y utilidad percibida como condiciones necesarias para un aprendizaje académico autorregulado. Anales de Psicología, 28(1), 37-44.

Rubio, M.J. y Galván, C. (2013). Portafolios digitales para el desarrollo de competencias transversales: Aportaciones principales de los estudios con Carpeta Digital en el marco del grupo de investigación Ensenyament i Aprenentatge Virtual. Digital Educational Review, 24, 53-68.

Rubio, M.J.; Galván, C. y RodríGuez, J.L. (2013). Propuesta didáctica para el uso de portafolios digitales en educación superior. EDUTEC (Revista Electrónica de Tecnologia Educativa), 43, 3-13. Recuperado de <http://www.edutec.es/revista/ index.php/edutec-e/article/view/334>.

Sánchez Santamaría, J. (2012). El e-portafolio en la docencia universitaria: Percepciones de los estudiantes y carga de trabajo. Revista Electrónica de Investigación y Docencia, 7. 
SAns, A. (2008). La evaluacón de los aprendizajes: Construcción de instrumentos. Barcelona: Graó.

Stefani, L.; Mason, R. y Pegler, C. (2007). The educational potential of e-portfolios. Londres: Routledge.

Stiggins, R. (2008). Assessment Manifesto: A coll for the development of balance assessment systems. Oregon: ETS Assement Training Institut.

TEJADA, J. (2006). El prácticum por competencias: Implicaciones metodológicoorganizativas y evaluativas. Bordón, 58(3), 121-139.

Te Wiata, I. (2001). A big ask: To what extent can universities develop useful generic skills? En F. Bevan, C. Kanes y D. Roebuck (eds.). Knowledge Demands for the New Economy (pp. 290-297). Brisbane: Australian Academic Press.

VÁsquez, S. (2011). Las comunidades de práctica. Educar, 47(1), 51-68.

Ventura, J. (2005). El Pràcticum en los estudios pedagógicos y la inserción laboral. Tesis doctoral presentada en la Universidad de Barcelona.

Vilà, R. y Aneas, A. (2013). Los seminarios de práctica reflexiva en el Prácticum de Pedagogía de la Universidad de Barcelona. Bordón, 65(3), 165-18. <http://dx.doi.org/10.13042/23171>

Vilà, R.; Aneas, A. y Rajadell, N. (2015). La evaluación de competencias del alumnado en las Prácticas Externas. Procedia - Social and Behavioral Sciences, 196(8), 226-232. <http://dx.doi.org/10.1016/j.sbspro.2015.07.034>

Vilà, R.; Burguet, M.; Aneas, A.; Rajadell, N.; Noguera, E. y Millan, D. (2014). El Pràcticum al grau de Pedagogia de la Universitat de Barcelona: El mapa de la professió, la pràctica simulada i la immersió en una organització. REIRE: Revista d'Innovació $i$ Recerca en Educació, 7(1), 11-29. $<$ http://dx.doi.org/10.1344/reire2014.7.1716>

Weinert, F. y Chapter, E. (2001). Concept of competence: A conceptual clarification. En D. Rychen y L. Salganik (ed.). Defining and selecting key competencies (pp. 45-65). Ashland, OH, US: Hogrefe \& Huber Publishers.

Wenger, E. (2001). Comunidades de práctica: Aprendizaje, significado e identidad. Barcelona: Paidós.

Wenger, E. y Sánchez-Barberán, G. (2001). Comunidades de práctica: Aprendizaje, significado e identidad. Barcelona: Paidós.

Wolters, C. (2003). Understanding procrastination from a self-regulated learning perspective. Journal of Educational Psychology, 95, 179-187.

Zabalza, M.A. (1998). El prácticum y las prácticas en empresas en la formación universitaria. En M.A. Zabalza y L. IgLesias (eds.). V Symposium Internacional sobre el Prácticum: Innovaciones en el Prácticum. Poio (Pontevedra): Universidad de Vigo.

Zimmerman, B.J. (2002). Becoming a Self-Regulated Learner: An Overview. Theory Into Practice, 41(2), 64-70.

Zubizarreta, J. (2009). The learning portfolio. San Francisco: Anker. 